Becker, T.; Windt, K.: A comparative view on existing autonomous control approaches - observations from a simulation study. In: Hülsmann, M.; Scholz-Reiter, B.; Windt, K. (eds.): Autonomous cooperation and control in logistics. SpringerVerlag, 2011. The original publication is available at www.springerlink.com.

\title{
A Comparative View on Existing Autonomous Control Ap- proaches - Observations from a Simulation Study
}

Till Becker, Katja Windt

\section{Abstract}

Autonomous control has been studied in detail in the recent years. Control algorithms have been developed along with strategical and technical solutions. Still there is lack of broad applications of autonomous control in industry, which impedes a comprehensive evaluation of autonomous control algorithms. The presented simulation study applies multiple control algorithms within a simulation model using real business data derived from a scenario of an automobile logistics company. The results point out which control methods are able to increase logistic targets achievement, but they also reveal where autonomous control reaches its limits.

\section{Introduction}

The concept of autonomous control offers additional possibilities to cope with fluctuating market conditions that have evolved over the recent years. Customers expect a variety of customized products and a fast product delivery, while a rising number of world-wide acting companies operate complex logistic networks around the globe (Scholz-Reiter et al. 2004). Such an interlinked network of logistic processes that belong to numerous companies lead to an enormous increase in dynamics and complexity regarding the ability to coordinate, control, and monitor these processes. Traditional production planning is likely to be disadvantageous in a situation that is characterized by high complexity and dynamic behavior. The reason can be found in the fact that an increase in complexity (e.g. number of links in a network, number of choices regarding variants) enables an exponential rise in possible combinations within a production program. Dynamic behavior affects production planning because changes in the environment occur while the "optimal" production program is still being calculated. This constant change of parameters, which are at the same time input parameters of the planning algorithm, renders the outcome of the planning process useless. Therefore, a different approach to complex and dynamic production planning problems is needed to overcome these two major obstacles.

Autonomous control in logistics is one possible answer to the problems of complexity and dynamics. It is "characterized by the ability of logistics objects to process information, to render and to execute decisions on their own" (Windt et al. 2008). Consequently, there is a spatiotemporal transfer of production planning decisions. Firstly, decision making now takes place within the logistic process itself, e.g. on the shop floor, instead of being carried out by a central department or a central IT system. Secondly, decisions are taken continuously during the process, ideally instantaneously before its execution in order to retain as much flexibility as possible (Windt and Jeken 2009). Classic production program planning realizes decision making prior to the execution of the complete production program, therefore having a clear temporal delay between decision making and decision execution.

Previous publications have already addressed the question whether there is a benefit for the application of autonomous control in producing companies (Windt et al. 2010a). Additionally, the mapping of specific autonomous control algorithms to specific areas of application has been investigated (Windt et al. 2010a). The present work will extent the scope of the previously published studies. It will shed light on the boundaries that companies are ex- 
pected to face when applying autonomous control in their logistic processes. The questions that are going to be addressed are as follows:

- Which autonomous control methods can be beneficial in different settings of production processes?

- What are the limitations of the investigated autonomous control methods regarding logistic targets achievement?

The following sections of this paper are guided by the two questions. Autonomous control methods including the algorithms behind autonomous control, are analyzed more thoroughly in the next section. The section "Simulation Model" contains a description of the simulation model that has been used to study the available autonomous control methods and their logistic performance, as well as a summary of the eight different control methods that have been implemented. In the section "Simulation Results", the experimental data is evaluated and interpreted in the context of the two research questions. Finally, the answers to the research questions are summarized in the "Conclusion" section.

\section{Autonomous Control Methods}

The introduction of autonomous control in a production logistics company has organizational, technological, and process-related demands (Scholz-Reiter et al. 2004). Organizational demands cover the need of autonomous logistic process description, the availability of local information, and more. Technical demands can be information processing ability of the autonomous actors, communication technology within the processes, and similar technology related requirements. Process-related demands include the actual control algorithms that determine how decision making is carried out. These algorithms are called autonomous control methods. They are defined according to the definition of autonomous control as "generic algorithms that describe how logistics objects render and execute decision by their own" (Windt et al. 2010b).

Autonomous control methods can have any degree of complexity regarding their decision making. A simple instance of an autonomous control method could allow semi-finished parts in a job-shop scenario to select the next step in their production by choosing the machine having the lowest number of items waiting in its queue (Scholz-Reiter et al. 2006). A more sophisticated method is inspired by ants' foraging behavior. A preferred path through the production is indicated by virtual pheromones that are emitted by successfully processed parts (Cicirello and Smith 2001).

Beside the work this paper builds upon (Windt et al. 2010a), there have been other analyses of autonomous control methods. Scholz-Reiter et al. (2009) conducted a simulation study as well, but compared only three different autonomous control methods. Furthermore, they focused on the logistic target achievement in distinct scenarios with varying process complexity and degree of autonomous control. Peng and Mcfarlane (2004) defined three different strategies for agent based manufacturing management. Their simulation was based on a general flow shop model. Their results indicate that going for the shortest waiting queue is a dominant solution compared to breakdown avoidance and proportional availability strategies. Other simulations (Scholz-Reiter et al. 2008a; Scholz-Reiter et al. 2008b) were utilized to prove the feasibility of newly developed autonomous control methods without a comparative aspect. A recent publication of Scholz-Reiter et al. (2010) investigates autonomous control in comparison to common scheduling heuristics. In contrast to the approach presented here, they utilized an exemplary production process model. 
A previous study of autonomous control methods has shown that the existing methods can be sorted in clusters which have common characteristics (Windt et al. 2010b). This has been so far the investigation that incorporated the largest number of methods in the area of autonomous control in logistics. Fourteen available methods have been categorized and evaluated in a simulation study. However, a simplified simulation model representing a job shop production scenario has been applied there. As the benefits and drawbacks of autonomous control methods in production logistic processes are brought into focus in this work, a more realistic simulation model has been used here. Furthermore, this model is derived from a real business case in production logistics. This yields to a higher confidence of the simulation results and their interpretation regarding the application of autonomous control methods in industry.

\section{Simulation Model}

\section{Model Description}

The simulation model designed for this study is based on the automobile terminal at the Hamburg port in Germany. The terminal is operated by the BLG LOGISTICS GROUP. The terminal is a transshipment point as well as a production site. In the highly standardized automotive industry car manufactures outsource non-standard modifications to contractors. The automobile terminal offers storage capacity for 12,000 cars on 324,000 square meters. Beside storage areas the terminal has technical facilities which allow the operator to conduct repairs, painting, washing, and technical modifications. The terminal receives cars via vessel, train, or truck. The distribution is usually done by truck.

In addition to the availability of the terminal data, the scenario has been selected for the simulation study due to the comparatively simple applicability of autonomous control. The processes on the terminal have a clear structure and all elements and actors are easily identifiable. The process-inherent flexibility regarding the sequence of the production steps enables autonomous, ad-hoc decisions. This process represents a class of multistage production processes.

The lifecycle of a car on the terminal starts with its arrival via vessel, train, or truck. All incoming cars are taken to the Incoming Deliveries Area. They are registered electronically according to their chassis number. The workers do a quick check and a damage survey if required. The car ID is linked to the orders which are already available in the IT system. Cars can have no orders at all and are supposed to be directly shipped to their final destination, which is usually a car dealer. Otherwise, there is a parking order and/or one or more treatment orders. When the receiving car dealer needs a car that is designated to him, he orders the car from the terminal. A retrieval order is created and the car is retrieved from storage, sent through the treatment if applicable, and placed on the Outgoing Deliveries Area. The necessary documents are created and the car leaves the terminal. A schematic layout of the terminal is displayed in Figure 1. 


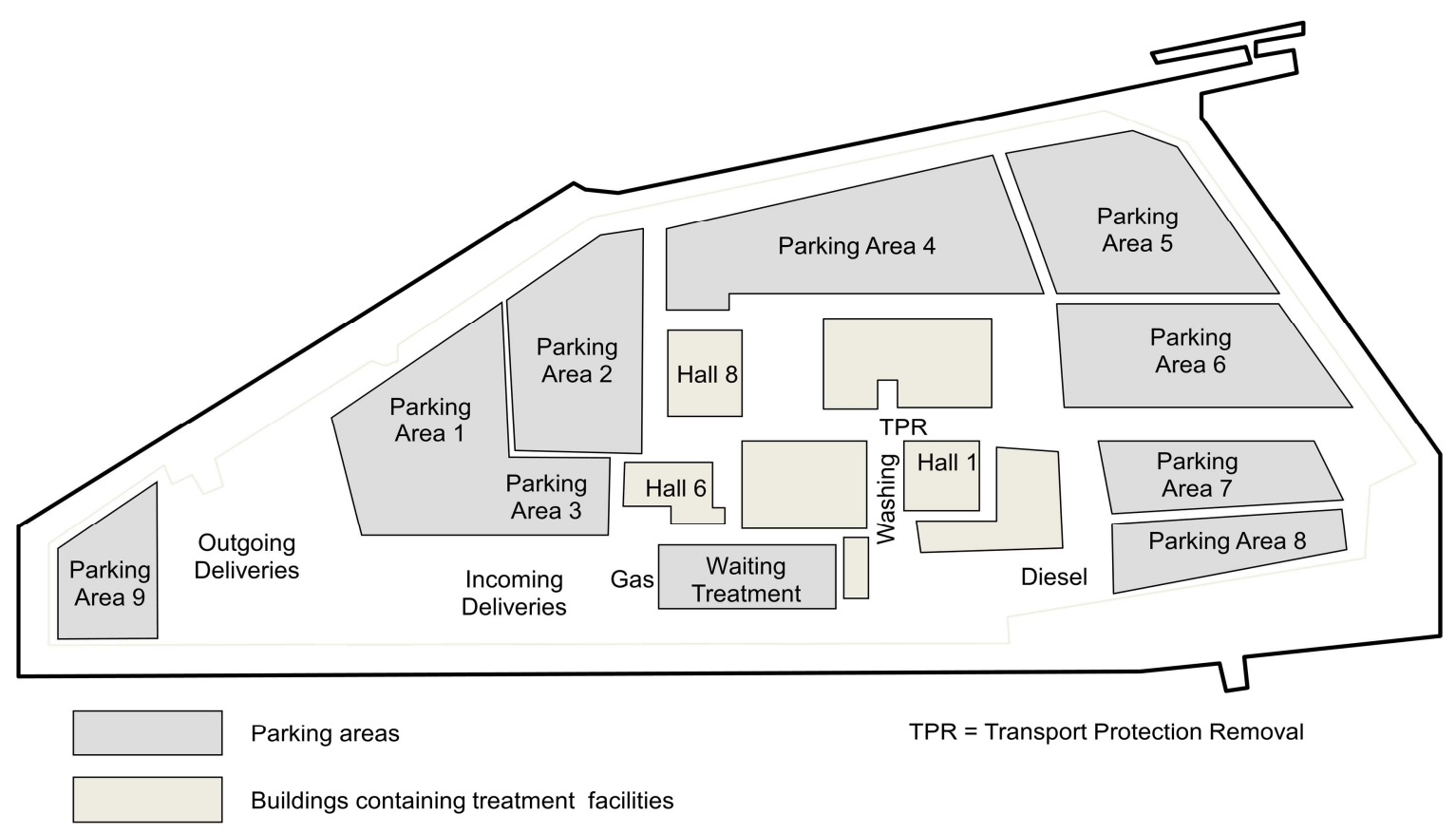

Figure 1: Schematic representation of the automobile terminal

In order to answer the two research questions, it is necessary to model the relevant aspects of the terminal's logistic processes as they take place in reality. For purposes of the simulation study, the control mechanism as it is used on the terminal is replaced by the selected autonomous control methods. Data from key figures is selected and evaluated according to the initial questions. The complete production data of the year 2008 serves as input data for the simulation study. The dataset includes 151,934 car records. Each record is composed of the arrival date, information on parking order including duration of parking, and a list of treatment orders.

The path of the virtual cars in the simulation model is depicted in Figure 2. All cars are registered at their arrival date at the Incoming Delivery Area. The process of transportation is not modeled explicitly as it has no relevance for the processing of the orders. Each car can either immediately be taken to the Outgoing Delivery Area, be transferred to one of the parking areas, or be transferred to the Treatment Waiting Area which serves as a buffer for all treatment stations. The choice depends on the existing orders for each individual car. Cars that are retrieved from a parking area either go to the Treatment Waiting Area if a treatment order is available or they go directly to the Outgoing Delivery Area and thus leave the simulation. Cars never return to the parking areas as treatment only takes place immediately before they are shipped. All cars that have at least one treatment order wait on the Treatment Waiting Area (buffer). After one single treatment has been finished cars enter again in the buffer if there are remaining unfinished treatment orders. Otherwise the car is transferred to the Outgoing Delivery Area.

The time for transferring a car from one point to the terminal to another is taken from a distance table. The table contains average values from all sources to each applicable destination. The treatment times are determined by randomly drawing a value from a table containing all treatment times for each station taken from the original dataset. Consequently, the treatment times in the simulation follow the same distribution as the times from the 2008 dataset. 


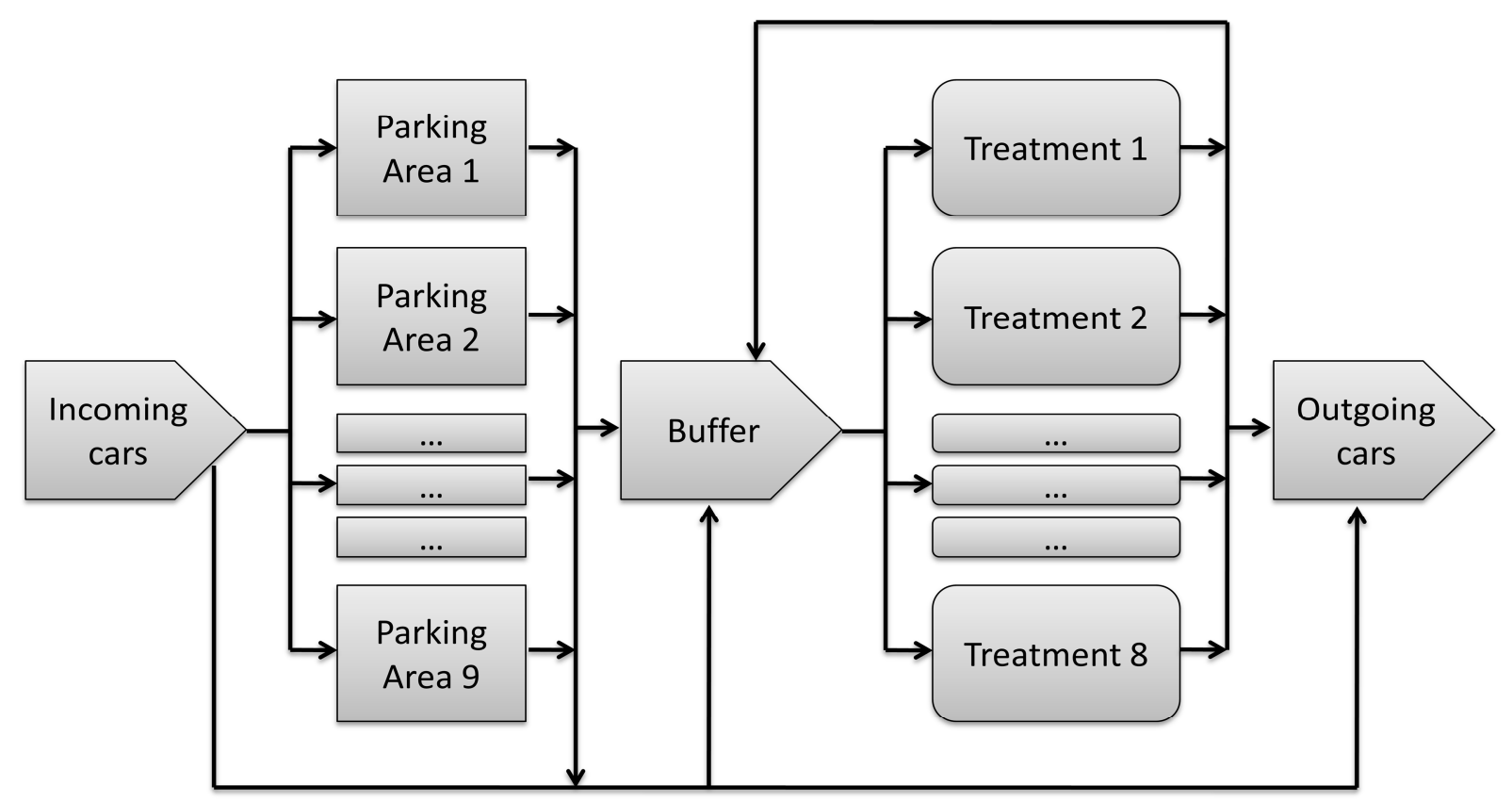

Figure 2: Possible paths of a car in the simulation model

\section{Autonomous Control Methods Applied}

The comparison of the different autonomous control methods is realized by embedding the control mechanism of each single method into the simulation model. For the selection of appropriate autonomous control methods, a thorough literature study has been performed. Control methods in the areas of autonomous control, self-control, multi-agent-based methods, smart systems, or decentralized control have been taken into consideration. There were two main criteria for the selection of a method: firstly, the methods need to be clearly documented so that it is possible to implement them in the simulation model. If the concept of a method is only described as a sketch, it cannot be guaranteed that it is implemented the way it is supposed to work. Secondly, the methods need to be applicable to the present simulation model. A too narrow scope of application (e.g. a restriction to transportation or assembly processes) would make the control methods useless for this scenario.

Autonomous control methods have been collected within the collaborative research center "Autonomous Cooperating Logistic Processes" (CRC637, see Acknowledgement Section) as well as from external sources. Table 1 gives an overview of the eight selected methods. The parameter "depth" which is given in the short description of the autonomous control method describes how many steps in advance are considered by the specific method (Windt et al. 2010c). E.g. a depth of two when using queue length as decision criterion indicates that the car would sum up the queue lengths of two following stations, thus creating a decision tree. Each decision alternative then consists of two consecutive treatments, valued by their summed queue lengths.

\begin{tabular}{|l|l|l|}
\hline Name & Source & Short description \\
\hline Standard (std) & $\begin{array}{l}\text { Automobile } \\
\text { terminal }\end{array}$ & $\begin{array}{l}\text { Fixed assignment of parking area } \\
\text { Fixed sequence of treatment steps }\end{array}$ \\
\hline DLRP (dlrp) & $\begin{array}{l}\text { Wenning et al. } \\
2007\end{array}$ & $\begin{array}{l}\text { Choose path with lowest estimated travel time to park- } \\
\text { ing / average travel + waiting + processing time; depth: } \\
\text { full }\end{array}$ \\
\hline
\end{tabular}




\begin{tabular}{|l|l|l|}
\hline $\begin{array}{l}\text { Ant Phero- } \\
\text { mone (ant) }\end{array}$ & $\begin{array}{l}\text { Scholz-Reiter } \\
\text { et al. 2008a }\end{array}$ & $\begin{array}{l}\text { After each car departs from a treatment station it leaves } \\
\text { a fixed amount of pheromones; } \\
\text { Pheromones add up with the already existing amounts at } \\
\text { the station; over time the pheromones slowly evaporate } \\
\text { Stations with higher pheromone levels are stations with } \\
\text { better throughput and are therefore preferred by the } \\
\text { cars; depth: 4 }\end{array}$ \\
\hline $\begin{array}{l}\text { Holonic (ho- } \\
\text { lonic) }\end{array}$ & $\begin{array}{l}\text { Van Brussel et } \\
\text { al. 1998 }\end{array}$ & $\begin{array}{l}\text { Choose path with lowest estimated travel time to park- } \\
\text { ing / average travel + waiting + processing time; depth: } 2\end{array}$ \\
\hline $\begin{array}{l}\text { Minimum } \\
\text { buffer (min- } \\
\text { buf) }\end{array}$ & CRC637 & $\begin{array}{l}\text { Choose parking area / treatment station with lowest oc- } \\
\text { cupation (relative buffer level); depth: 2 }\end{array}$ \\
\hline $\begin{array}{l}\text { Queue Length } \\
\text { Estimation } \\
\text { (qle) }\end{array}$ & CRC637 & $\begin{array}{l}\text { Choose path with lowest queue length (\# of items); } \\
\text { depth: 1 }\end{array}$ \\
\hline $\begin{array}{l}\text { Random (ran- } \\
\text { dom) }\end{array}$ & CRC637 & $\begin{array}{l}\text { Random assignment of parking area } \\
\text { Random sequence of treatment steps }\end{array}$ \\
\hline $\begin{array}{l}\text { Simple rule } \\
\text { based (sr2) }\end{array}$ & $\begin{array}{l}\text { Scholz-Reiter } \\
\text { et al. 2006 }\end{array}$ & $\begin{array}{l}\text { Choose path with lowest estimated travel time to park- } \\
\text { ing / waiting and processing time; depth: 4 }\end{array}$ \\
\hline
\end{tabular}

Table 1: Autonomous control methods applied in the simulation study. Parameter depth indicates how many following processing steps are taken into consideration for the decision (full means that all remaining steps were evaluated). (Windt et al. 2010a)

The standard (std) method describes a set of rules which is based on the procedures that have been applied at the automobile terminal at the time when processes and data have been recorded. The parking areas are filled in a specific order. The order depends on the existence of one or more treatment orders for a car. Cars without treatment orders are assigned to different parking areas. All treatment orders, if available, are executed in a fixed sequence. Tables 2 and 3 show the priority lists applied in the simulation for the standard method.

\begin{tabular}{|c|l|l|}
\hline Priority & With treatment & Without treatment \\
\hline 1 & Parking Area 4 & Parking Area 1 \\
\hline 2 & Parking Area 5 & Parking Area 3 \\
\hline 3 & Parking Area 6 & Parking Area 2 \\
\hline 4 & Parking Area 7 & Parking Area 4 \\
\hline 5 & Parking Area 8 & Parking Area 5 \\
\hline 6 & Parking Area 9 & Parking Area 6 \\
\hline 7 & Parking Area 2 & Parking Area 7 \\
\hline 8 & Parking Area 3 & Parking Area 8 \\
\hline 9 & Parking Area 1 & Parking Area 9 \\
\hline
\end{tabular}

Table 2: Priority list parking areas

\begin{tabular}{|l|l|}
\hline Rank & Treatment Station \\
\hline 1 & Gas or Diesel \\
\hline 2 & Transport Protection Removal \\
\hline
\end{tabular}




\begin{tabular}{|l|l|}
\hline 3 & Washing \\
\hline 4 & Hall 6 \\
\hline 5 & Hall 8 \\
\hline 6 & Hall 1 \\
\hline
\end{tabular}

Table 3: Priority list treatment stations

Another special method is the random method. This method is not an autonomous control method in the sense of a specific strategy. It simply chooses the following step (parking area or treatment station) by random selection. The selection follows a uniform distribution over the number of alternatives available, not considering the capacity. The two methods, standard and random, have been implemented in the simulation in order to let the autonomous control methods compete against them.

\section{Data Evaluation Scheme}

A performance evaluation can only be done if a key figure measurement is available. The four targets of production logistics (short throughput time, high due date reliability, high utilization, and low work in process) can serve as a measurement system in many logistic applications (Nyhuis and Wiendahl 2008). In the simulation model throughput time has been measured in separate portions. They consist of travelling time, parking time, and treatment time. As the real due dates could not be derived from the available dataset, the due date for each car has been set to 4 working days after its retrieval date. The retrieval date is recorded in the original dataset. It is the point in time when the customer (usually the retailer) demands the car. At that point a car is transferred initially to the treatment area, either from the parking area or directly from the incoming area. Both throughput time and due date reliability are based on actual working shifts, excluding nights, weekends, and holidays. Utilization as well as inventory levels of parking areas and treatment stations have been collected on a daily basis. Work in process as a key figure has been left out because due to the static input data the average work in process over the whole year remains unchanged, regardless of the control method.

Although it would be difficult to draw conclusions about exact performance values of the automobile terminal from the simulation study, the values for throughput and travel times have been multiplied with a hidden factor to protect the company's business data. The due date reliability as it is calculated here is a realistic assumption but the individual due dates are part of non-public contracts of the company. The specific due date reliability figures in this study do not necessarily need to match the companies true due date reliability. However, the approach used here allows for a judgment of due date reliability relations between the tested methods.

\section{Simulation Model Parameters}

The terminal model and the given input data for the year 2008 represent a fixed environment in which the different autonomous control methods have been investigated. But one important point of autonomous control and self-organization is the fact that the methods are meant to adapt to different situations. On the one hand, this happens over time depending on the varying arrival of cars over the year. On the other hand, one of the initial questions in this work aims at getting a better understanding under which circumstances a specific autonomous control methods performs better or worse. Therefore, additional simulation runs with a variation of selected model parameters have been carried out. The scenar- 
io as it has been described up to now is called the basic scenario. This includes the presented model and the original 2008 production data.

The second scenario is called "full flexibility" (Windt et al. 2010d). In this setting the restrictions regarding the sequence of treatment orders have been suspended. Consequently, the average number of decision alternatives for each car increases, resulting in a higher flexibility of the products. Although this does not represent the actual situation in this real world application of autonomous control methods, it gives a hint how the performance of the methods changes under varying flexibility.

A third scenario investigates the methods' performance depending on the workload. In this "increased load" scenario the treatment times have been increased by $10 \%$. Consequently, the capacity of the whole system is reduced. Finally, a fourth scenario combines both modifications and includes higher flexibility and increased workload.

For each combination of the four scenarios with each of the control methods, five independent simulation runs have been carried out in order to ensure the significance of the results. Statistical tests have been applied to verify the significance of the deviations between the figures presented in the next section. The error values have not been included in the histograms due to the high spread of the individual values. This spread, however, is not caused by an arbitrariness of the model. It is a result of the vast variation in the input data between the individual cars regarding the number of orders, the individual treatment times, and the fluctuating workload over the year.

\section{Simulation Results}

\section{Utilization}

The simulation results will be presented according to the previously mentioned production logistics targets. One of the targets is to keep utilization of the resources at a high level. Resources in the simulation model are the parking areas, which correspond to storage facilities on a more generic level. The automobile service provider seeks to fully utilize its parking areas, because the customers pay fees for the storage of their cars on a daily basis. The treatment stations can be seen as machines or assembly stations in terms of adding value to a product. Only the parking area utilization is presented here, because due to the fixed structure of the input data (fixed number of cars, fixed assignment of treatment orders) there was no variation in the treatment station utilization figures. Furthermore, the scenario modifications did not affect parking area utilization, so that only results from the standard scenario are presented here.

Figure 3 shows a graph indicating the average parking area utilization in the simulation model over the whole year. The methods ant and qle have not been included in the utilization evaluation as these methods are not applicable to the parking area selection decision. The virtual pheromone approach used by the ant method is depending on the evaporation of the pheromones over time. However, the time a car spends on the parking area is externally determined and therefore the evaporation would not represent waiting time as the parking time biases the decision variable pheromone level. The qle method bases its decisions on the lengths if waiting queues occur. The lack of waiting queues at the parking areas renders the decision method useless at this point. In the simulation, the parking decisions for these two methods have been substituted by the minbuf method. 


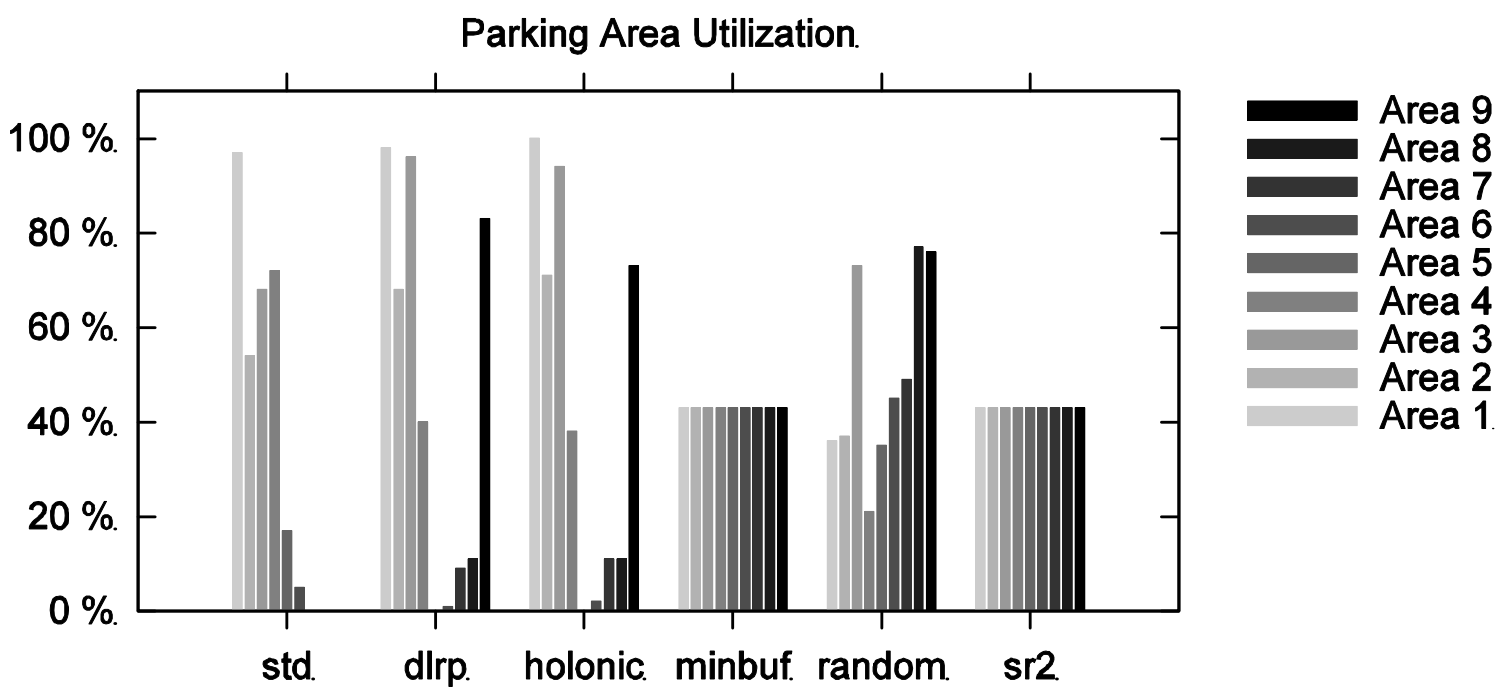

Figure 3: Average parking area utilization on the automobile terminal (adapted from Windt et al. 2010a).

There are different characteristic patterns of parking area utilization that can be observed in Figure 3. The dlrp and holonic method have almost the same distribution pattern of utilization, because they consider the same variables for their decision. Minbuf and sr 2 distribute the cars equally between all storage areas, as these methods select the parking area with lowest utilization and therefore level the utilization over all areas. At first glance it seems surprising that random does not distribute equally. The answer is that random makes an equally distributed choice between all parking areas, which can have different capacities. Consequently, high capacity parking areas are less utilized and vice versa. Std directs cars to the parking areas following the priority list given in Table 2.

\section{Throughput Time: Travel}

The average times recorded for travelling to and from the parking area are displayed in Figure 4. Again, the methods ant and qle which cannot make parking relevant decisions are left out. Values are shown for the basic scenario due to lack of significant differences between the different scenarios. It can be clearly seen that dlrp and holonic outperform the other approaches in terms of travel times. The below-average performance of minbuf, ran$d o m$, and sr2 are the trade-off for leveling the utilization of all parking areas, which also includes more distant parking sites. 


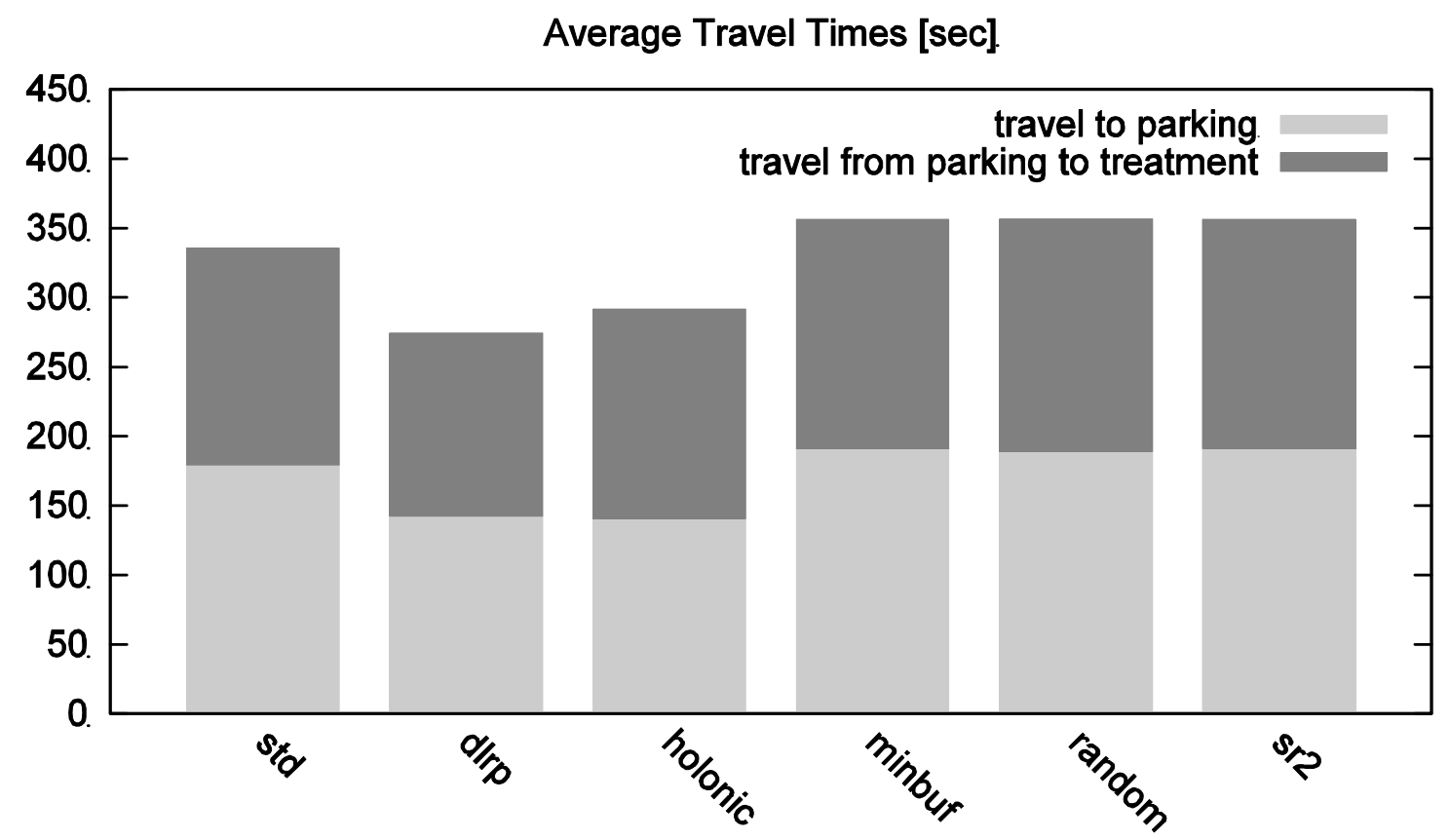

Figure 4: Average travel times from the incoming area to parking and from parking to treatment (adapted from Windt et al. 2010a).

\section{Throughput Time: Treatment}

In the basic scenario (Figure 5, grey colums) all methods except minbuf manage to outperform the simple std method regarding average throughput time. Curiously, even the random method delivers higher throughput time performance. If random is selected as benchmark, dlrp, holonic, and sr2 remain with increased performance. Interestingly, sr2 as a less sophisticated decision method in comparison to dlrp and holonic delivers the best result. This conveys the impression that in this scenario the average values used by dlrp and holonic do not match the actual situation exactly enough. The overall picture changes a bit when looking at the full flexibility scenario. The increased number of decision alternatives enables ant and qle to outperform the other methods. The information that is expressed by the virtual pheromone level in the ant method seems to reflect best the situation in the production process. Std remains unchanged due to the fixed allocation of order sequence, while random performs worst. 


\section{Average Treatment Times [min]}

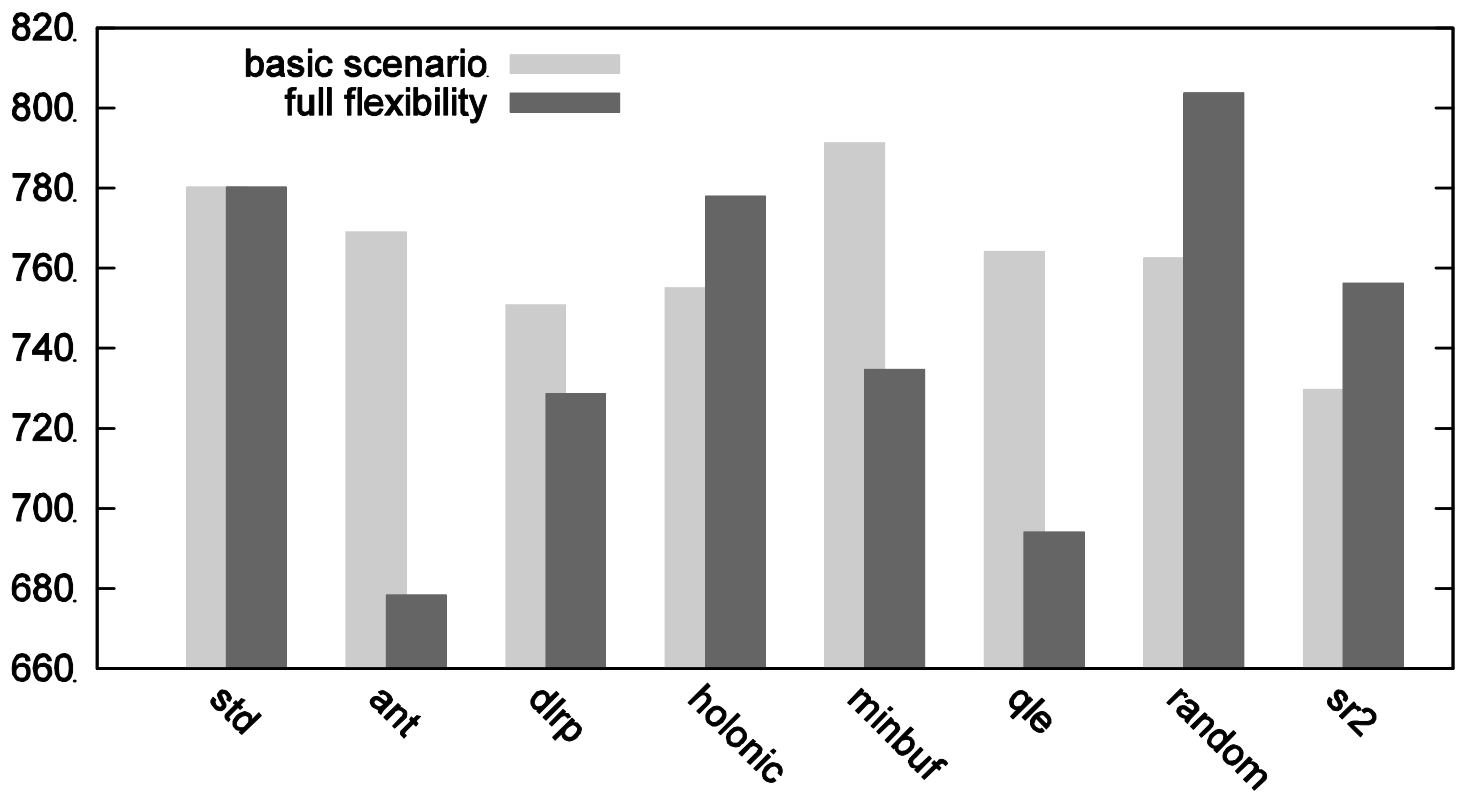

Figure 5: Average treatment times of all cars that have at least on treatment order (basic scenario and full flexibility scenario).

Figure 6 depicts the average treatment times in the increased load and the combined scenario. Results are somehow similar for the increased load scenario. In the case of a combination of increased load and full flexibility, ant and dlrp are the only methods that can outperform the std approach. Again, the virtual pheromone figure seems to offer a good indicator for throughput time decisions. Both dlrp and ant perform significantly better in a scenario with higher flexibility.

Average Treatment Times [min] - Increased Load

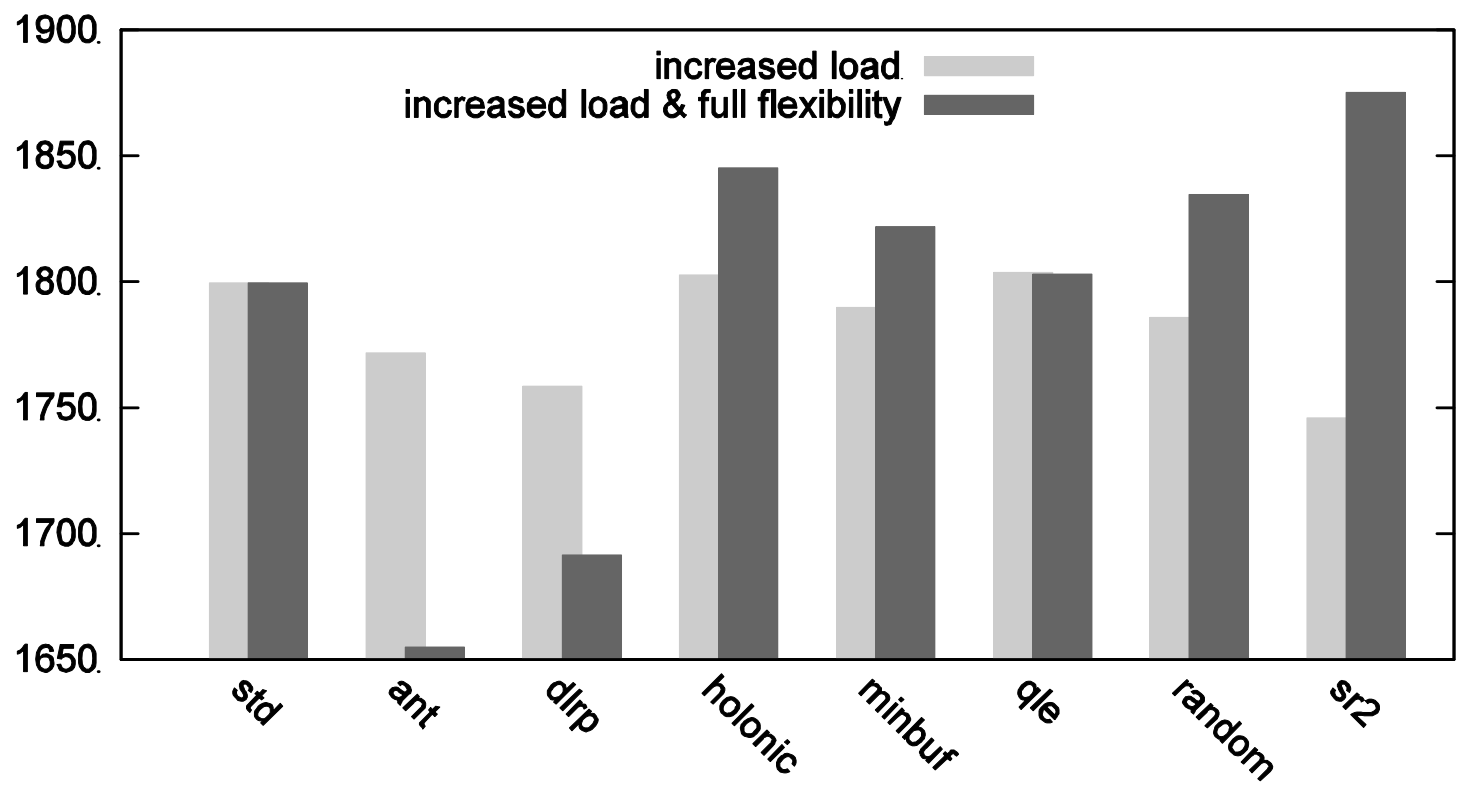

Figure 6: Average treatment times of all cars that have at least on treatment order (increased load only and increased load \& full flexibility scenario). 


\section{Due Date Reliability}

Due date reliability can be increased by any method in any scenario in comparison to the std approach. The difference among the methods decreases coming from the basic scenario to full flexibility (Figure 7). Due date reliability drops in general under increased load (Figure 8). Adding more flexibility lets the majority of the autonomous control methods perform better.

Average Due Date Reliability.

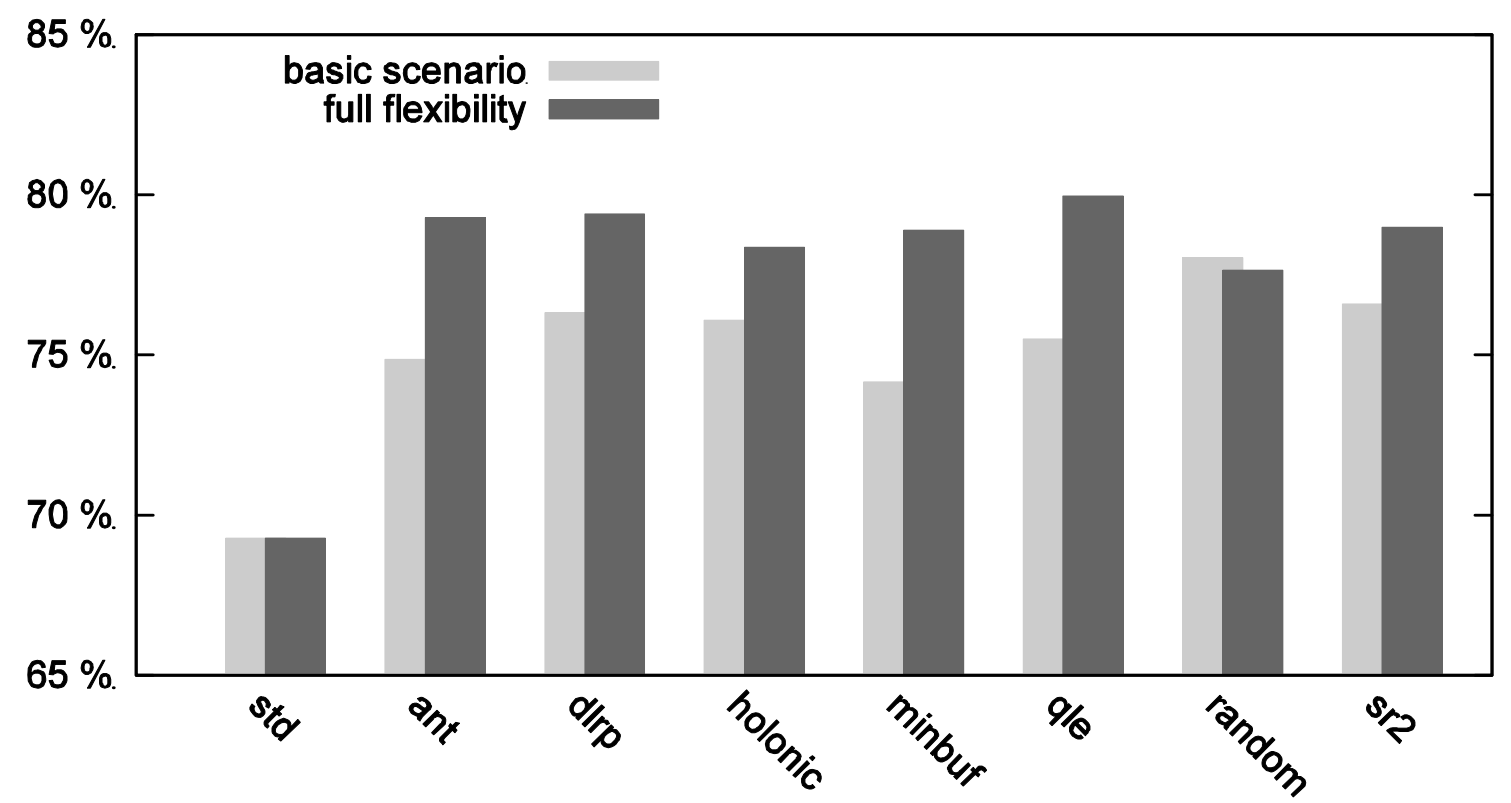

Figure 7: Average due date reliability (basic scenario and full flexibility scenario).

Average Due Date Reliability - Increased Load.

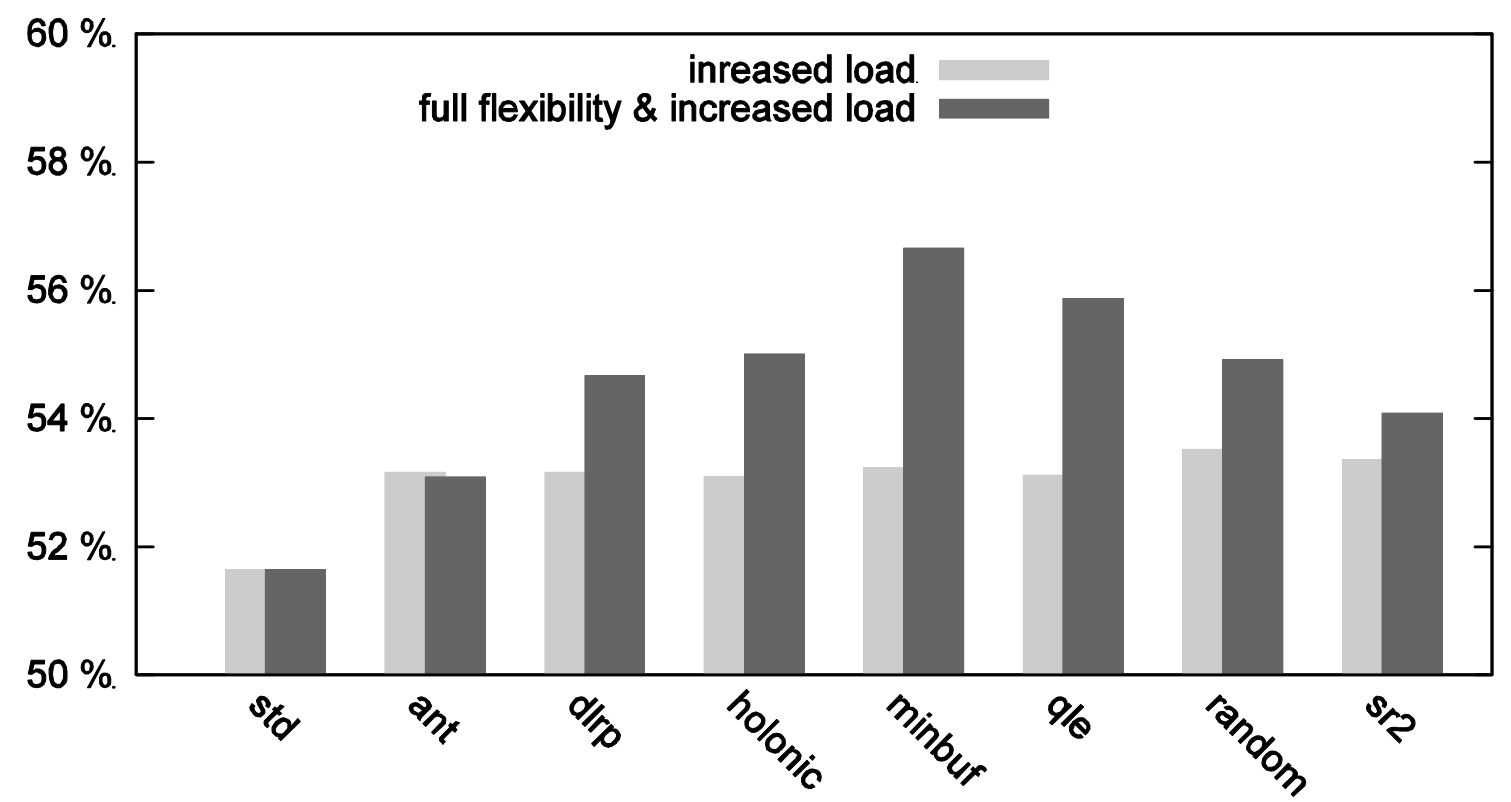

Figure 8: Average due date reliability (increased load only and increased load \& full flexibility scenario). 


\section{Opportunities and Limitations of Autonomous Control}

The simulation study delivered diverse results regarding the performance of autonomous control methods in a production logistics application scenario. First of all it has to be stated that there is no single autonomous control method that dominates all other methods in the achievement of all logistic targets in any scenario.

A holistic view on the parking process (representing storage process in production logistics) includes the results from parking area utilization and travel times to and from parking. DIrp and holonic manage to reduce travel times significantly and should be preferred among investigated methods for the purpose of storage. If the major goal is the balanced utilization of storage capacities, minbuf and $s r 2$ perform best.

The production (treatment) part needs more differentiation. The results vary depending on the available flexibility in the production process and the workload of the system. In general, dlrp has a good overall performance. However, in specific scenarios it can be significantly outperformed (e.g. by ant in full flexibility scenarios). Therefore, the results tend to suggest a distinct selection of the autonomous control algorithm, depending on various parameters, including logistic target preference, number of decision alternatives, and workload of the system.

Limitations of autonomous control can be seen from two perspectives: firstly, the presented figures indicate that autonomous control methods can perform worse than static planning methods or even a random decision making. Consequently, autonomous control does not inherently outperform other control approaches. A second perspective that might limit the application of autonomous control is the difficulty of selecting the appropriate autonomous control method. There is no single dominant method that can be applied in an arbitrary scenario. The introduction of autonomous control needs a careful judgment and customization. A possible solution can be the distinguished consideration of autonomous control methods for sub processes or different system states (e.g. high workload vs. low workload). Furthermore, a single autonomous control method could be parameterized to adapt itself to a changing environment.

Finally it needs to be stated that this investigation used an inductive approach. The performance of the selected autonomous control methods has been observed in one specific scenario that represents a class of multistage production processes. Although the automobile terminal scenario has been carefully selected and implemented, there may be different results for the same autonomous control methods in distinct scenarios.

\section{Conclusion}

This work has presented a simulation study based on a real world logistics scenario. Several autonomous control methods have been applied in the simulation and their performance regarding different logistic key figures has been analyzed. The results clearly point out that the application of autonomous control has to be customized for each individual scenario. The parameters of the logistic environment as well as logistic target preference determine which autonomous control method to use. It is also conceivable that different autonomous control methods are applied interchangeably in the same scenario, depending on surrounding conditions.

Future research should aim at developing a clear structure for the classification of autonomous control methods regarding their parameters. In combination with additional simulation experiments in more diverse scenarios it will be possible to indentify the highperformance methods with greater certainty. 


\section{Acknowledgement}

This research is funded by the German Research Foundation (Deutsche Forschungsgemeinschaft, DFG) as the Collaborative Research Center 637 "Autonomous Cooperating Logistic Processes - A Paradigm Shift and its Limitations". 


\section{Bibliography}

Cicirello V, Smith S (2001) Ant colony control for autonomous decentralized shop floor routing. In: ISADS-2001, Fifth International Symposium on Autonomous Decentralized Systems

Nyhuis P, Wiendahl H (2008) Fundamentals of Production Logistics: Theory, Tools and Applications, Springer Verlag, Berlin Heidelberg

Peng Y, Mcfarlane D (2004) Adaptive Agent-based Manufacturing Control and its Application to Flow Shop Routing Control, Production Planning and Control, 15(2):145-155

Scholz-Reiter B, Freitag M, de Beer C, Jagalski T (2006) The Influence of Production Network's Complexity on the Performance of Autonomous Control Methods. In: Teti R (ed) Intelligent Computation in Manufacturing Engineering, Proceedings of the 5th CIRP International Seminar on Computation in Manufacturing Engineering (CIRP ICME '06), University of Naples, Naples, pp 317-320

Scholz-Reiter B, Görges M, Philipp T (2009) Autonomously Controlled Production Systems - Influence of Autonomous Control Level on Logistic Performance. CIRP Annals of Manufacturing Technology, 58(1):395-398

Scholz-Reiter B, Windt K, Freitag M (2004) Autonomous logistic processes: New demands and first approaches. In: Monostori, L. (ed.): Proc. 37th CIRP International Seminar on Manufacturing Systems, Hungarian Academy of Science, Budapest, Hungaria, pp. 357-362

Scholz-Reiter B, Freitag M, de Beer C, Jagalski T (2006) The Influence of Production Networks' Complexity on the Performance of Autonomous Control Methods, 5th CIRP International Seminar on Computation in Manufacturing Engineering (CIRP ICME '06), Proceedings of the 5th CIRP International Seminar on Computation in Manufacturing Engineering (CIRP ICME '06), University of Naples, Naples, pp 317-320

Scholz-Reiter B, de Beer C, Freitag M, Jagalski T (2008a) Bio-inspired and Pheromonebased Shop-floor Control, International Journal of Computer Integrated Manufacturing, 1/2:201-205

Scholz-Reiter B, Jagalski T, Bendul (2008b) Autonomous Control of a Shop Floor Based on Bee's Foraging Behavior, In: Proceedings of the First International Conference on Dynamics in Logistics (LDIC), pp 415-423

Scholz-Reiter B, Rekersbrink J, Görges M (2010) Dynamic flexible flow shop problems Scheduling heuristics vs. autonomous control, CIRP Annals of Manufacturing Technology,

Volume 59(1):465-468

Van Brussel H, Wyns J, Valckenaers P, Bongaerts L, Peeters P (1998) Reference Architecture for Holonic Manufacturing Systems: PROSA, Computers in Industry, 37/3:255-274

Wenning B-L, Rekersbrink H, Timm-Giel A, Görg C, Scholz-Reiter B (2007) Autonomous Control by Means of Distributed Routing. In: Hülsmann M, Windt K (eds.): Understanding Autonomous Cooperation \& Control in Logistics, Springer, Berlin Heidelberg, pp 325-335

Windt K, Böse F, Philipp T (2008) Autonomy in Production Logistics - Identification, Characterisation and Application, Int J Robotics CIM 24(4):572-578

Windt K, Jeken O (2009) Allocation Flexibility - a New Flexibility Type as an Enabler for Autonomous Control in Production Logistics. In: 42nd CIRP Conference on Manufacturing Systems, Grenoble

Windt K, Becker T, Kolev I (2010a) A comparison of the Logistics Performance of Autonomous Control Methods in Production Logistics, Sihn, W.; Kuhlang, P. (eds.): Proceedings of the 43rd CIRP International Conference on Manufacturing Systems. Sustainable Production and Logistics in Global Networks, NW Verlag, Vienna, pp 576-583 
Windt K, Becker T, Jeken O, Gelessus A (2010b) A Classification Pattern for Autonomous Control Methods in Logistics, Logistics Research: Volume 2, Issue 2 (2010), pp 109-120

Windt K, Becker T, Asenov D, Arbabzadah F (2010c) A Generic Implementation Approach of Autonomous Control Methods in Production Logistics. In: Proceedings of the Eighth IEEE International Conference on Control \& Automation, Xiamen, China, 9.-11.6.2010, pp 629-633

Windt K, Jeken O, Becker T (2010d) Selbststeuerung in der Produktion - Verbesserte Logistikleistung durch Ausschöpfung von Flexibilitätspotentialen in Fertigung und Montage. In: ZWF Zeitschrift für wirtschaftlichen Fabrikbetrieb, 105(2010)5, pp 439-443 